\title{
Innovative Approaches to Remote Sensing in NASA's Earth System Science Pathfinder (ESSP) Program
}

Frank Peri ${ }^{\mathrm{a}}$, Dr. Stephen Volz ${ }^{\mathrm{b}}$,

${ }^{a}$ NASA Langley Research Center, 9 Langley Boulevard, Hampton, VA, USA 23681;

bNASA Headquarters, 300 E Street SW, Washington, DC, USA 20024

\begin{abstract}
NASA's Earth Venture class (EV) of mission are competitively selected, Principal Investigator (PI) led, relatively low cost and narrowly focused in scientific scope. Investigations address a full spectrum of earth science objectives, including studies of the atmosphere, oceans, land surface, polar ice regions, and solid Earth. EV has three program elements: EV-Suborbital (EVS) are suborbital/airborne investigations; EVMission (EVM) element comprises small complete spaceborne missions; and EV-Instrument (EVI) element develops spaceborne instruments for flight as missions-of-opportunity (MoO). To ensure the success of EV, the management approach of each element is tailored according to the specific needs of the element.
\end{abstract}

Keywords: ESSP, Earth Venture, program management

\section{INTRODUCTION}

Following recommendations of the National Research Council Decadal Survey in 2007, NASA's Earth System Science Pathfinder Program (ESSP) introduced the Earth Venture class (EV) of mission opportunities. The ESSP is a strategic investment by NASA's Science Mission Directorate (SMD), Earth Science Division (ESD) that includes a series of relatively low-to-moderate cost, small-to-medium sized, competitively selected, PI led missions that are built, tested, and launched in a short time interval to accommodate new and emergent scientific priorities. ESSP projects support a variety of earth science objectives, including the atmosphere, oceans, land surface, polar ice regions, and solid Earth. The investigations in the project complement the larger, strategic missions identified by name in the Decadal Survey and provide flexibility to accommodate scientific advances and new implementation approaches. The projects encompass the entire life cycle from definition, through design, development, integration and test, launch or deployment, operations, science data analysis and distribution. The ESSP Program Office, located at Langley Research Center (LaRC), is responsible for the management, direction, and implementation of the ESSP program elements.

\section{PROGRAM GOALS AND OBJECTIVES}

ESSP Program goals and objectives trace to NASA's needs, goals, and objectives. The 2011 NASA Strategic Plan specifies six Strategic Goals for the Agency. The 2010 Science Plan: For NASA's Science Mission Directorate (SMD Science Plan) details how SMD will turn NASA's science vision into scientific discovery. The Science Plan identifies six Earth Science Research Program Science Focus Areas: atmospheric composition, weather, carbon cycle and ecosystems, water and energy cycle, climate variability and change, and Earth surface and interior.

The goal of the ESSP Program is to stimulate new scientific understanding of the global Earth system through the development and operation of remote-sensing missions and the conduct of investigations utilizing data from these missions to address unique, specific, highly focused requirements in Earth science research.

The ESSP Program objectives to achieve this goal are to:

- provide frequent periodic opportunities for competitively selected, PI-led projects addressing NASA's high priority Earth system science outcomes

- contain project and mission costs through commitment to, and control of, design, development, and operational costs within the risk and technical standards established by the Agency 
ESSP projects pursue science investigations in one or more of the six Earth Science Research Program Science Focus Areas and promote the outcomes listed above. By addressing the Science Focus Areas in innovative ways, the Earth Science community can understand variability, forcing, and response mechanisms from new perspectives. ESSP provides flexible opportunities to stimulate new scientific understanding by encouraging increased participation by small projects and creativity in all aspects of project development; the implementation of these lead to new strategies for acquiring and distributing datasets. ESSP projects also demonstrate measurement techniques for application on future Earth Science operational missions.

ESSP organizational objectives are defined that guide the implementation approach for the program. They are to:

- tailor the management approach of the different program elements (suborbital, orbital, instrument) consistent with the cost, schedule, technical and risk objectives of that element.

- perform assessments of ESSP projects and use lessons learned and best practices to take action to ensure success.

- promote decision making based upon clearly established cost, schedule, technical and risk parameters for each project.

\section{PROGRAM ARCHITECTURE}

The ESSP projects have independent science objectives, mission requirements, and/or technical interdependencies, and yet are integrated in the program through a common funding and management structure. The projects may provide synergist coincident science measurements with other NASA projects (ESSP or non-ESSP) that enhance the overall science return.

EV has three program elements: EV Suborbital (EVS) are suborbital/airborne investigations with 5-year duration and managed to NASA Procedural Requirement (NPR) 7120.8. The EV Mission (EVM) element comprises small complete missions launched within 5 years of initiation, managed as Class D missions per NPR 7120.5, and cost-capped at $\$ 150 \mathrm{M}$. The EV Instrument (EVI) element develops spaceborne instruments for flight as missions-of-opportunity (MoO). Instruments are managed according to Class $\mathrm{C}$ per NPR 7120.5, limited to 5-year development duration and cost capped at \$90M. NASA separately secures and funds the access to space for these instruments. Table 3-1 summarizes the EV mission elements.

\begin{tabular}{|l|l|l|l|l|l|}
\hline & \multicolumn{1}{|c|}{ Duration } & $\begin{array}{c}\text { Cost Cap } \\
(\mathbf{F Y 1 4 \$})\end{array}$ & $\begin{array}{c}\text { Governing } \\
\text { NPR }\end{array}$ & \multicolumn{1}{|c|}{$\begin{array}{c}\text { Mission } \\
\text { Class }\end{array}$} & \multicolumn{1}{c|}{$\begin{array}{c}\text { Call } \\
\text { Frequency }\end{array}$} \\
\hline EV Suborbital & $\leq 5$ years & $<30 \mathrm{M}$ & 7120.8 & N/A & 4 years \\
\hline EV Mission & $\leq 5$ years to launch & $<150 \mathrm{M}$ & $7120.5 \mathrm{E}$ & Class D & 4 years \\
\hline EV Instrument & $\leq 5$ years to delivery & $<90 \mathrm{M}$ & $7120.5 \mathrm{E}$ & Class C & $15-18$ months \\
\hline
\end{tabular}

Table 3-1 Earth Venture Mission Types

Table 3-2 describes all the ESSP projects and their current status.

\begin{tabular}{|l|l|l|l|l|}
\hline \multicolumn{1}{|c|}{ Project } & \multicolumn{1}{c|}{ Date } & Category/Class & \multicolumn{1}{c|}{ Phase } & \multicolumn{1}{c|}{ Status } \\
\hline Orbital Missions & Launch & & & \\
\hline GRACE & March 2002 & $3 / *$ & Phase E & Extended Operations \\
\hline CALIPSO & April 2006 & $3 / *$ & Phase E & Extended Operations \\
\hline CloudSat & April 2006 & $3 / *$ & Phase E & Extended Operations \\
\hline Aquarius & June 2011 & $2 / \mathrm{C}$ & Phase E & Operations \\
\hline OCO-2 & July 2014 & $2 / \mathrm{C}$ & Phase C & Implementation \\
\hline OCO-3 & August 2016 & $3 / \mathrm{C}$ & Phase A & Formulation \\
\hline EVM-1: CYGNSS & 2016 & $3 / \mathrm{D}$ & Phase A & Formulation \\
\hline EVI-1: TEMPO & TBD & $\mathrm{C}$ & Phase A & Formulation \\
\hline
\end{tabular}




\begin{tabular}{|l|l|l|l|l|}
\hline Sub-Orbital & First Deployment & & & \\
\hline EVS-1: AirMOSS & June 2012 & N/A & Phase E & Operations \\
\hline EVS-1: ATTREX & September 2011 & N/A & Phase E & Operations \\
\hline EVS-1: CARVE & June 2011 & N/A & Phase E & Operations \\
\hline EVS-1: DISCOVER-AQ & Jun 2011 & N/A & Phase E & Operations \\
\hline EVS-1: HS3 & Aug 2012 & N/A & Phase E & Operations \\
\hline
\end{tabular}

* Category/class was not defined during mission implementation

Table 3-2: ESSP Project Portfolio

\section{MISSION ELEMENT SPECIFIC MANAGEMENT APPROACH}

To ensure the success of the ESSP Program, the management approach of each EV element is tailored according to the specific characteristics of the element. The following sections define each program element.

\subsection{EV-SUBORBITAL}

The EV Suborbital (EVS) element are suborbital, competitively selected, PI led investigations to conduct innovative, integrated, hypothesis, or scientific question driven approaches to pressing earth science issues.

Earth Venture Suborbital investigations have the following characteristics:

- Sustained, science-based data acquisition - Investigations advance earth science objectives through temporally sustained regional- or larger-scale measurements sufficient and necessary to prove/disprove a scientific hypothesis or address scientific questions.

- Mature technology - Investigations must use mature system technology where, at a minimum, there has been a system/subsystem model or prototype demonstration in a relevant environment.

- Cost and schedule constraints - Each suborbital Venture-class investigation must have a life cycle duration of less than or equal to five years with total investigation cost not to exceed $\$ 30$ million.

\subsubsection{Principal Investigator Responsibility}

An EVS PI is wholly responsible to accomplish the investigation objectives using his/her own management processes, procedures, and methods. The PI is responsible for all planning and documentation for the investigation, including science goals and objectives, baseline and threshold science requirements and investigation implementation approach.

\subsubsection{Risk Management}

For EVS investigations the PI identifies potential risks to successful achievement of investigation objectives within resource and schedule constraints. The PI specifies risk mitigation plans, including descopes if appropriate. Generally, the nature of suborbital investigations allows for an aggressive risk posture compared to spaceflight missions. For example, investigations occur during deployments of equipment to a field location with a schedule established for taking measurements that has some margin, i.e. extra days built in. If any equipment exhibits anomalous operations or failure, the PI's team can, in some cases, perform repairs on the equipment without impacting the deployment schedule. As such, subsystem redundancy is not required, for example.

\subsubsection{NASA Oversight}

The PI has a large degree of freedom and responsibility to accomplish the science objectives. NASA's oversight ensures that the investigation is responsive to requirements and the constraints of NPR 7120.8 for the risk that is acceptable for suborbital investigations. 


\subsubsection{Reviews}

EVS investigations have a minimal, streamlined review structure that includes the following events: Investigation Confirmation Review (ICR)

The ICR generally occurs within one year of investigation selection after the PI and ESD agree upon investigation requirements, the PI matures the investigation implementation approach, and the major risks to completion of the proposed investigation have been addressed. The original proposal forms the baseline for the assessment. Following the assessment, the PI presents their investigation and responses to the assessment to ESD. The ICR will be complete when the ESD approves the investigation to continue with implementation.

Flight Readiness Review (FRR)/Operations Readiness Review (ORR)

The FRR/ORR is conducted according to NASA policies and procedures to ensure the instrumentation and aircraft are ready and safe for flight. This review does not generally assess the performance of the instrumentation.

\section{Project Status Review (PSR)}

PSRs are conducted quarterly by the investigation team and ESSP to examine the progress to date against the approved cost, schedule, and performance of the investigation.

\section{Science Review}

The purpose of the Science Review is to measure progress toward meeting the baseline and threshold requirements listed in the PLRA and is conducted at least annually.

\subsection{EV-MISSION}

The Earth Venture Missions (EVM) element are spaceflight missions defined to conduct innovative integrated, hypothesis or scientific question-driven approaches to pressing earth science issues. They are PI led and encompass all measurements required to achieve the scientific objectives. The Associate Administrator of the SMD is the Decision Authority and is supported by the Directorate Program Management Council that recommends approval or disapproval to the Decision Authority for entry to the next phase at Key Decision Points (KDP).

\subsubsection{Principal Investigator Responsibility}

An EVM PI is responsible for accomplishing the proposed mission objectives. The PI defines the technical implementation and the project management approach that drives the risk posture. The PI can exercise decisions to accomplish requirements within a trade space that includes performance margins, quality assurance, and reliability. The PI is responsible for defining and describing in the formulation agreement (Phase $\mathrm{A} / \mathrm{B}$ ) or project plan (Phase $\mathrm{C} / \mathrm{D} / \mathrm{E} / \mathrm{F}$ ) the standards, processes and practices for mission assurance, the mission implementation (approach \& execution), the approach for performance/cost/schedule/risk management and the approach for peer reviews.

\subsubsection{Risk Management}

Because EVM projects can be classified as Class D, the risk tolerance is typically higher than other earth science missions (most of which are Class C). The PI implements a rigorous and accountable risk management process that identifies any risk that is accepted (rather than mitigated). EVM projects are allowed to proceed to launch with some unmitigated yellow risks (those with likelihood greater than 3 ).

\subsubsection{NASA Oversight}

The PI has a large degree of freedom and responsibility to accomplish the proposed science objectives and implement the mission. NASA's oversight ensures that the project is performing to applicable standards. While Class D missions are required to comply with the requirements of NPR 7120.5, NPR 7123, the PI may propose to tailor NASA processes or use their institution's processes. NASA exercises only essential oversight to ensure implementation is responsive to requirements and constraints of NPR 7120.5 for the risk that are acceptable for Class D implementations. 


\subsubsection{EVM Life Cycle Reviews}

EVM reviews will be conducted as specified in NPR 7120.5 and NPR 7123.1. As a Category 3 activity with LCC $<\$ 250 \mathrm{M}$, an independent review team is established to conduct the design reviews that precede KDP events. EVM mission reviews (except KDP's) are led by a NASA Center. For missions selected that are not led by a NASA Center, LaRC will serve as the host NASA center with responsibility to satisfy Agency Technical Authority requirements.

\subsubsection{Expectations to achieve a successful Class D Mission}

The successful implementation of a Class D mission requires acceptance of principles and practices by a community that traditionally expects a lower risk tolerance than that allowed on Class D missions. In order to be successful, different constitutes are champions of the Class D management approach. The following are some key ways the constituents contribute to success:

\section{$\underline{\text { Stakeholders }}$}

- Ensure the Class D characteristics are applied to all reviews and evaluations

- Ensure the cost cap criteria of the program is maintained as a parameter of the project

\section{NASA Headquarters (ESD) and Program Office}

- Maintain Class D risk posture through launch

- Encourage innovative implementations

- Maintain vigilance against requirements creep and risk suppression

- Encourage innovative implementations

Principal Investigator

- Keep open communications on implications of Class D risk management process and mission implementation

- Recognize that termination for excessive cost is real

\subsection{EV-INSTRUMENT}

The Earth Venture Instrument (EVI) element develops instruments for participation on a NASA-arranged spaceflight mission of opportunity to conduct innovative, integrated, hypothesis or scientific questiondriven approaches to pressing earth science issues. The NASA funded PI retains a central role on the instrument or instrument package development, integration and testing, calibration, and science operations. The Associate Administrator of the SMD is the Decision Authority and is supported by the Directorate Program Management Council that recommends approval/disapproval to the Decision Authority regarding entry to next phase at Key Decision Points.

\subsubsection{Principal Investigator Responsibility}

An EVI PI is responsible for accomplishing the proposed mission objectives. The PI defines the technical implementation and the project management approach that drives the risk posture. The PI can exercise decisions to accomplish requirements within a trade space that includes performance margins, quality assurance, and reliability. The PI is responsible for defining and describing in the formulation agreement (Phase $\mathrm{A} / \mathrm{B}$ ) or project plan (Phase $\mathrm{C} / \mathrm{D} / \mathrm{E} / \mathrm{F}$ ) the standards, processes and practices for mission assurance, the mission implementation (approach \& execution), the approach for performance/cost/schedule/risk management and the approach for peer reviews. 
Costs that are within the PI Managed responsibility:

- Instrument development

- Functional algorithms and ground processing system

- Science team

- Calibration/validation activities

- Operations, product generation, data analysis

- Key management and engineering staff during Phase D

Costs that are outside the PI Managed responsibility:

- Integration to NASA selected platform

- Required funding to cover the gap between instrument delivery and start of platform I\&T

- Access to space

\subsubsection{Risk Management}

For EVI projects the risk tolerance is consistent with typical earth science projects that are Class C. The PI will implement a rigorous and accountable risk management process that identifies any consequences of risk that are accepted. The PI identifies the risks and the mitigations while NASA examines consistency with the risk tolerance for a Class $\mathrm{C}$ instrument development.

\subsubsection{NASA Oversight}

The PI has a large degree of freedom and responsibility to accomplish the proposed science objectives. NASA's oversight ensures that the project is performing to applicable standards. NASA uses its standard policy and processes to evaluate the PI established management processes to ensure the rigor required for success. NASA exercises only essential oversight to ensure project implementation is responsive to requirements and constraints of NPR 7120.5 for the risk that are acceptable for Class C implementations.

\subsubsection{EV-I Life Cycle Reviews}

EV-I reviews will be conducted as specified in NPR 7120.5 and NPR 7123.1. As a Category 3 activity with LCC less than $\$ 250 \mathrm{M}$, an independent review team is established in place of an IPAO SRB. EV-I reviews (except KDP's) will be led by a NASA Center. For missions selected that are not led by a NASA Center, the LaRC will serve as the host NASA center with responsibility to satisfy Agency Technical Authority requirements.

The PI can propose tailored technical reviews subject to approval by ESD. An independent review team is established by the lead NASA Center. The chair of the review team reports findings to the PI, lead NASA Center, Program Office, and HQ. The Independent review team is involved in major reviews and not dayto-day implementation.

\subsubsection{Expectations to achieve a successful "Instrument-First" Mission}

The success of the EVI element hinges on the necessity to mitigate the most critical instrument development risks prior to making an external commitment with stakeholders on the life-cycle cost and launch date. In doing so, NASA hopes to avoid large cost overruns that have challenged the Agency in recent years.

\section{SUMMARY}

With diverse mission approaches, acute risk awareness and a steady tempo of new mission selections, NASA's Earth Venture missions are positioned to continue NASA's preeminence in world-class earth system science. Program management approaches tailored to the specific risk posture, implementation approach and science objectives will enable cost effectiveness and science performance to drive NASA's decisions. 